\title{
Exploiting kinematics and UBVI $c$ photometry to establish high fidelity membership of the open cluster Blanco 1
}

\author{
P. A. Cargile ${ }^{1}$, D. J. James ${ }^{1}$, I. Platais ${ }^{2}$, J.-C. Mermilliod ${ }^{3}$, C. \\ Deliyannis $^{4}$ and A. Steinhauer ${ }^{5}$ \\ ${ }^{1}$ Department of Physics and Astronomy, Vanderbilt University \\ Nashville, TN 37235, USA \\ email: p.cargile@vanderbilt.edu \\ ${ }^{2}$ Johns-Hopkins University, Baltimore, MD, USA \\ ${ }^{3}$ EPFL Lausanne, Lausanne, Switzerland \\ ${ }^{4}$ Universtiy of Indiana, Bloomington, IN, USA \\ ${ }^{5}$ SUNY-Geneseo, Geneseo, NY, USA
}

\begin{abstract}
We present the results of a wide-field, high-precision $U B V I_{c}$ CCD photometric survey of the Galactic open cluster Blanco 1. Standardized photometry was acquired using the Y4Kcam on the SMARTS $1 \mathrm{~m}$ telescope at CTIO. We have also determined new high-precision proper motions $\left(\sigma_{\mu}=0.3\right.$ mas $\left.y^{-1}\right)$ over an eight square degree area down to $V=16.5$. Combined with $1 D$ kinematic data, our survey yields a complete list of cluster members down to $\sim 0.5 M_{\odot}$ and new high-fidelity color-magnitude diagrams are presented for Blanco 1 . Having established a bona fide membership catalog, astrophysical characteristics of solar-type cluster members such as X-ray activity and lithium abundance have been studied to gain more insights in the process of internal mixing and convection. Our new results should also help to better understand its peculiar location in the Milky Way and to unravel its dynamical history.
\end{abstract}

Keywords. astrometry, techniques: photometric, open clusters and associations: individual (Blanco 1)

\section{Introduction}

Blanco 1 is a rather poor, young $(50-100 \mathrm{Myr})$, and nearby $(240 \mathrm{pc})$ open cluster. It is unique among open clusters because of its unusually high Galactic latitude $\left(b=-79^{\circ}\right)$, especially for its age. Blanco 1 also shows strong evidence, due to its Galactic latitude and kinematics, that it has passed through, and even interacted with, the Galactic disk. We present here the results of a wide-field, high-precision $U B V I_{c}$ CCD photometric survey of Blanco 1, including new high-fidelity color-magnitude diagrams for the cluster. Moreover, we have determined new high-precision proper motions $\left(\sigma_{\mu}=0.5\right.$ mas $\left._{y^{-1}}{ }^{-1}\right)$ over an eight square degree area down to $V=16.5$.

\section{Observations and results}

The astrometric reductions are based on 32 sets of photographic plates and CCD frames almost all of them obtained with the $51 \mathrm{~cm}$ double astrograph $\left(\mathrm{scale}=55^{\prime} \mathrm{mm}^{-1}\right)$ at Cesco Observatory in El Leoncito, Argentina. These observations span 40 years ending in September 2007. Proper motions and positions are calculated using a variant of the central plate-overlap method (e.g, Herbig \& Jones 1981) and the UCAC2 catalog (Zacharias et al. 2004) as a reference frame. The precision of our proper motions, for stars 


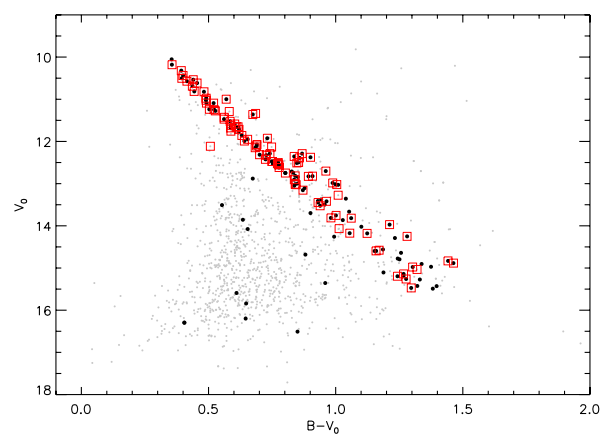

(a)

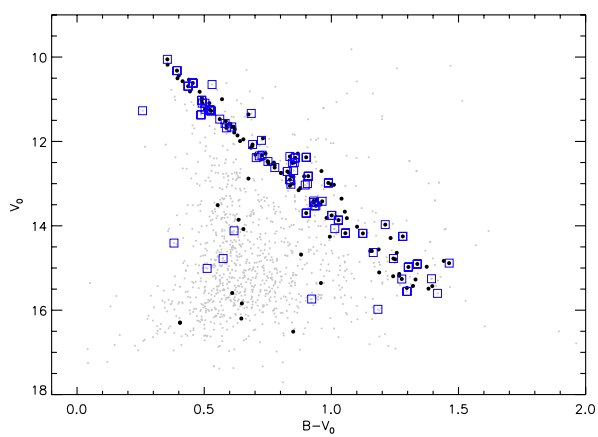

(b)

Figure 1. $V$ vs. $B-V$ CMD for Blanco 1 with proper motion members identified (bold points). (a) Objects with radial velocities consistent with the systemic velocity of the cluster are identified (red boxes). (b) Optical counterparts to x-ray sources are identified (blue boxes).

with optimal image properties, is 0.3 mas $y^{-1}$. The final catalog contains 6300 objects, among which, more than 3600 have proper-motion precisions better than 2 mas $_{\mathrm{yr}^{-1}}$.

The formal proper-motion membership probabilities $P_{\mu}$ were calculated using the probability definition formulated by Vasilevskis et al. (1958): $P_{\mu}=\Phi_{c} / \Phi_{c}+\Phi_{f}$ where $\Phi_{c}$ is the distribution of cluster stars, and $\Phi_{f}$ is the distribution of field stars in the vectorpoint diagram. We used the so-called local sample method to derive the field and cluster distributions, which works very well in sparse open clusters (Platais et al. 2007). The separation between the cluster and field is convincing for the entire magnitude range and a total of 247 stars have their $P_{\mu}$ greater than $0 \% . U B V I_{c}$ CCD photometric data were taken using the SMARTS 1.0m telescope at the CTIO, equipped with the $19.3 \mathrm{arcmin}^{2}$ Y4K camera. The central $1.6 \times 1.3$ sq. degrees of Blanco 1 were surveyed in a $5 \times 4$ grid mosaic (see James et al. 2008, in prep).

In Fig. 1 we plot the $V$ vs. $B-V$ CMD for Blanco 1 with proper motion members identified. The majority of astrometric members of the cluster lie along a well defined main-sequence locus. In Fig. 1a, it is clearly seen that stars identified with radial velocities consistent with the systemic velocity of the cluster trace the same main-sequence as the proper motion members (see James et al. 2008; Mermilliod et al. 2008). In Fig. 1b, we now identify the optical counterparts to objects with X-ray emission, which can be used as a membership criterion in young clusters (Cargile \& James 2008). The vast majority of the X-ray sources in Blanco 1 trace the same cluster sequence as defined by both the proper motion and radial velocity data.

\section{References}

Cargile, P. A. \& James, D. J. 2008, $A J$, in prep

Herbig, G. H. \& Jones, B. F. 1981, AJ, 86, 1232

James, D. J., et al. 2008, $A J$, in print

Mermilliod, J-C., et al. 2008, $A \mathscr{G} A$, in prep

Platais, I., et al. 2007, A\&SA, 461, 509

Vasilevskis, S., Klemola, A., \& Preston, G. 1958, AJ, 63, 387

Zacharias, N., Urban, S. E., Zacharias, M. I., Wycoff, G. L., Hall, D. M., Monet, D. G., \& Rafferty, T. J. 2004, AJ, 127, 3043 